\title{
Efecto del parasitismo en campo de Billaea Claripalpis Wulp. criadas en diatraea Saccharalis Fabr. y Galleria Mellonella L.
}

Effect of parasitism in the field of Billaea Claripalpis Wulp. maids in diatraea Saccharalis Fabr. and Galleria Mellonella L.

${ }^{1}$ Charles Frank Saldaña Chafloque ${ }^{a}$

\section{RESUMEN}

Determinar el efecto del parasitismo en campo de Billaea claripalpi sobre Diatraea saccharalis, criadas en su huésped natural Diatraea saccharalis, comparándolo con el hospedero alterno Galleria mellonella en Virú, La Libertad. El análisis de varianza muestra diferencias significativas entre los tratamientos, cabe mencionar, que Billaea claripalpis criadas en su huésped artificial Galleria mellonella, tratamientos T1 y T4, son las que obtuvieron mejores promedios de parasitación; seguidas por los tratamientos T3 y T2, en ambos tratamientos Billaea claripalpis fueron criadas en su huésped natural Diatraea saccharalis. En la cosecha, la relación del porcentaje de intensidad de infestación es inversamente proporcional al porcentaje de parasitismo, con un coeficiente de correlación de $\mathrm{r}=-0.97$ y con $\mathrm{R}^{2}=0.9479$, a modo que incrementa el porcentaje de control de Billaea claripalpis, decrece el porcentaje de intensidad de infestación, demostrando que este parasitoide es responsable de la disminución de los daños de Diatraea saccharalis en el fundo. Billaea claripalpis criadas en su huésped artificial Galleria mellonella, son las que obtuvieron mejores promedios de parasitación sobre Diatraea saccharalis. Es importante evaluar a nivel de laboratorio los diferentes agentes potenciales de control biológico, antes de iniciar un programa de masivo debido a la alta especificidad en parasitoides y agentes entomopatógenos.

Palabras clave: parasitismo, Billaea claripalpis, Galleria mellonella, Diatraea saccharalis, caña de azúcar,

\begin{abstract}
In this investigation the objective was to determine the effect of field parasitism of Billaea claripalpis on Diatraea saccharalis, maids in its natural host Diatraea saccharalis, comparing it with the alternate host Galleria mellonella in Virú, La Libertad. The analysis of variance shows significant differences between the treatments, it is worth mentioning that Billaea claripalpis maids in its artificial host Galleria mellonella, treatments T1 and T4, are the ones that obtained the best parasitic averages; followed by treatments $\mathrm{T} 3$ and $\mathrm{T} 2$, in both treatments Billaea claripalpis were raised in their natural host Diatraea saccharalis. In the harvest, the ratio of the percentage of infestation intensity is inversely proportional to the percentage of parasitism, with a correlation coefficient of $r=-0.97$ and with $\mathrm{R} 2=0.9479$,
\end{abstract}


as the percentage of Billaea claripalpis control increases, the Infestation intensity percentage, demonstrating that this parasitoid is responsible for decreasing the damage of Diatraea saccharalis in the place. Billaea claripalpis maids in their artificial host Galleria mellonella, are the ones that obtained the best parasitic averages on Diatraea saccharalis. It is important to evaluate at the laboratory level the different potential biological control agents, before starting a massive program due to the high specificity in parasitoids and entomopathogenic agents.

Keyword: parasitism, Billaea claripalpis, Galleria mellonella, Diatraea saccharalis, sugarcane

${ }^{1}$ Universidad Nacional Autónoma de Tayacaja Daniel Hernández Morillo, Pampas, Tayacaja, Huancavelica - Perú.

${ }^{\text {a } B i o ́ l o g o ~}$ 


\section{INTRODUCCIÓN}

Existe una numerosa variedad de insectos plaga que afectan la caña de azúcar y, excepto algunas especies que son cosmopolitas, tienen una distribución geográfica limitada y han pasado de otras gramíneas a alimentarse de la caña de azúcar (Cadena, 2008). El principal representante de este grupo se encuentran algunas especies de barrenadores, siendo Diatraea saccharalis Fabr. (Lepidoptera: Pyralidae), la más importante especie barrenadora y de importancia económica que ataca al cultivo de caña de azúcar, en América (Risco, 1960; Long y Hensley, 1972; Cadena, 2008; Salvatore et al 2009; Bustillo, 2009)

El método de control biológico de Diatraea saccharalis se ha basado en la liberación de controladores biológicos como Billaea (Paratheresia) claripalpis Wulp. (Diptera: Tachinidae), que en su estado de larva parasita las larvas del barrenador y se alimenta de ellas provocando la reducción de la población de plaga, por consiguiente, su daño. (Rodríguez, 2015)

En el Perú, específicamente en el norte del país, como La Libertad, Ancash, existen empresas dedicadas a la producción de Billaea claripalpis a partir de la utilización de su huésped natural Diatraea saccharalis Fabr. y de hospedante alterno como Galleria mellonella L., fácil de criar bajo condiciones controladas y de asepsia. Por tanto, para obtener una cría eficiente de un 10 Rev. Tayacaja 2(2): $08-18,2019$ ISSN:2617-9156 insecto se requiere conocer su ciclo de vida en función de la humedad y la temperatura, con el fin de establecer las condiciones óptimas para un proceso de producción masiva en laboratorio. (Rodríguez, 2015)

Galleria mellonella L, llamada también polilla grande la cera, los adultos ponen huevos en las celdillas de las colmenas. Las larvas salen entre tres y cinco días después, siendo muy activas y poseen un aparato masticador muy potente, y con él hacen túneles y galerías que envuelven con unas redes de seda, pueden viajar a colonias vecinas, y se ha comprobado que pueden llegar a recorrer 50 metros. Cuando los adultos salen del capullo se reproducen y comienzan el ciclo con la puesta de huevos que lleva a cabo la hembra (Goodman, 2003). Además, Neira (2006) indica que el ciclo de Galleria mellonella varía de acuerdo a las condiciones de temperatura, en uno o dos meses con temperaturas optimas que van desde los 26 a $38^{\circ} \mathrm{C}$, o cinco meses con temperaturas más bajas. Presentan los siguientes estados de desarrollo de huevo, larva, pupa y adulto.

El ciclo de vida de Diatraea saccharalis con presenta algunas diferencias en su duración, como resultado de la nutrición de la planta hospedera, condiciones ambientales, sobre todo la temperatura y la humedad relativa. Las larvas pasan a través de seis instars, de las cuales el primero ocurre sobre su superficie foliar y los demás dentro el tallo de la planta 
hospedera. Esta plaga presenta cuatro generaciones durante seis meses, pasando cada generación por los estados de desarrollo de huevo, larva, pre pupa, pupa y adulto, la duración de las generaciones oscila entre 41,3 días a 51,6 días, en diferentes tipos de clima. (Cornejo et al 2010)

En esta investigación el objetivo principal fue determinar el efecto del parasitismo en campo de Billaea claripalpis Wulp. sobre Diatraea saccharalis, criadas en su huésped natural Diatraea saccharalis Fabr., comparándolo con el hospedero alterno Galleria mellonella L. en Virú, La Libertad.

\section{MATERIALES Y MÉTODOS}

\section{Lugar de ejecución}

El presente trabajo presento una etapa de laboratorio y otra en campo, siendo el primer lugar en el Laboratorio de la Empresa SANIDAD VEGETAL TRUJILLO S.A.C., ubicada en el Distrito de La Esperanza, Provincia de Trujillo, Departamento de la Libertad y el segundo lugar, en el Fundo Santa Elena I, campo de cultivo de caña de azúcar, ubicada en las coordenadas geográficas de $8^{\circ} 24^{\prime} 55.9^{\prime \prime}$ Latitud Sur y 78 $45^{\prime} 7.2^{\prime \prime}$ Longitud Oeste, con una superficie de 24.09 has, a una altitud de 75 m.s.n.m., en el Sector de Santa Elena, Distrito de Virú, Provincia de Virú, Departamento de la Libertad.

\section{Material Biológico}

El material biológico para la obtención de adultos de Billaea clariplapis; fueron larvas de su huésped natural Diatraea saccharalis, y larvas del hospedero alterno Galleria mellonella. El material biológico fue proporcionado por el Laboratorio de la Empresa SANIDAD VEGETAL TRUJILLO S.A.C., la misma que a continuación describe la técnica de crianza utilizada para la obtención adultos de Billaea clariplapis, larvas de Diatraea saccharalis y Galleria mellonella.

\section{Técnicas de crianza}

\section{Crianza Galleria mellonella}

Se realizó según la técnica de Castillo, 2011, a partir de adultos se obtuvieron los huevos que se llevaron a un recipiente de plástico de 250 g.con una capa delgada de $5 \mathrm{~g}$ de polen, en donde los huevos eclosionaron y las larvas de primer instar continuaron alimentándose del polen. En el segundo instar, éstas fueron llevadas a otros recipientes de plástico de $500 \mathrm{ml}$ para ser alimentadas de manera artificial hasta su quinto instar. El alimento utilizado fue preparado utilizando alimento para "perro" marca Dog Chow, miel de azúcar y afrecho de trigo.

\section{Crianza Diatraea saccharalis Fabr.} Se realizó la crianza según las técnicas de Risco et al, 1973; Ayquipa y Sirlopú, 1978; Calderón, 1986; Pollack y Ayquipa, 1992; Mostacero, 2003 y Limo, 2009.

\section{Crianza Billaea claripalpis Wulp.}

Se realizó la crianza masiva de Billaea claripalpis a partir de larvas parasitadas Wulp., proporcionadas por la Empresa 
SANIDAD VEGETAL TRUJILLO

S.A.C., hasta la obtención de larvas, que fueron utilizadas para los experimentos. (Ayquipa y Sirlopú, 1978).

Parasitación de larvas de Diatraea saccharalis y Galleria mellonella

A los 18 días del acondicionamiento y apareamiento, las moscas grávidas de Billaea claripalpis se disectaron para extraer el ovisaco, de donde se obtuvo las cresas que fueron utilizadas para la parasitación, tanto de larvas de Diatraea saccharalis como de Galleria mellonella, posteriormente fueron colocadas en tambores de cedazo. Este proceso de parasitación se realizó bajo la técnica de parasitación masiva. (Pollack y Ayquipa, 1992).

\section{Liberación de Billaea claripalpis}

Se procedió con la liberación de la mosca nativa Billaea claripalpis, en el Fundo Santa Elena I, campo de cultivo de caña de azúcar, variedad CC87-434, caña planta, en una superficie total del experimento de 24.09 has., ubicada en el Sector de Santa Elena, Distrito de Virú, Provincia de Virú, Departamento de la Libertad (tabla 1), realizándose la liberación de la mosca nativa al sexto mes de edad del cultivo, según cada tratamiento ( tabla 2).

Tabla 1. Información del campo de cultivo de caña de azúcar, según tratamiento y repeticiones.

\begin{tabular}{cccccccc}
\hline Fundo & Cuartel & $\begin{array}{c}\text { Área } \\
\text { (ha) }\end{array}$ & $\begin{array}{c}\text { Fecha de } \\
\text { siembra }\end{array}$ & Variedad & $\begin{array}{c}\text { Tipo } \\
\text { caña }\end{array}$ & Tratamiento & Repetición \\
\hline Santa Elena I & 1 & 0.99 & $16 / 04 / 2017$ & CC.87-434 & Planta & T1 & R1 \\
Santa Elena I & 2 & 0.99 & $16 / 04 / 2017$ & CC.87-434 & Planta & T1 & R2 \\
Santa Elena I & 3 & 2.09 & $18 / 04 / 2017$ & CC.87-434 & Planta & T1 & R3 \\
Santa Elena I & 4 & 2.09 & $18 / 04 / 2017$ & CC.87-434 & Planta & T1 & R4 \\
Santa Elena I & 5 & 2.22 & $18 / 04 / 2017$ & CC.87-434 & Planta & T2 & R1 \\
Santa Elena I & 6 & 2.22 & $18 / 04 / 2017$ & CC.87-434 & Planta & T2 & R2 \\
Santa Elena I & 7 & 2.26 & $18 / 04 / 2017$ & CC.87-434 & Planta & T2 & R3 \\
Santa Elena I & 8 & 2.26 & $18 / 04 / 2017$ & CC.87-434 & Planta & T2 & R4 \\
Santa Elena I & 9 & 2.25 & $18 / 04 / 2017$ & CC.87-434 & Planta & T3 & R1 \\
Santa Elena I & 10 & 2.25 & $18 / 04 / 2017$ & CC.87-434 & Planta & T3 & R2 \\
Santa Elena I & 11 & 0.55 & $19 / 04 / 2017$ & CC.87-434 & Planta & T3 & R3 \\
Santa Elena I & 12 & 0.55 & $19 / 04 / 2017$ & CC.87-434 & Planta & T3 & R4 \\
Santa Elena I & 13 & 0.69 & $19 / 04 / 2017$ & CC.87-434 & Planta & T4 & R1 \\
Santa Elena I & 14 & 0.69 & $19 / 04 / 2017$ & CC.87-434 & Planta & T4 & R2 \\
Santa Elena I & 15 & 1.02 & $19 / 04 / 2017$ & CC.87-434 & Planta & T4 & R3 \\
Santa Elena I & 16 & 1.02 & $19 / 04 / 2017$ & CC.87-434 & Planta & T4 & R4 \\
Total & & $\mathbf{2 4 . 0 9}$ & & & & & \\
\hline
\end{tabular}


Tabla 2. Tratamientos empleados en las evaluaciones de parasitismo de Billaea claripalpis, según el huésped de crianza.

\begin{tabular}{ccc}
\hline Tratamiento & $\begin{array}{c}\text { Billaea claripalpis criada } \\
\text { en huesped }\end{array}$ & $\begin{array}{c}\text { Dosis } \\
\text { (parejas/ } \\
\text { ha) }\end{array}$ \\
\hline T1 & Galleria mellonella & $50 \mathrm{par} / \mathrm{ha}$ \\
$\mathrm{T} 2$ & Diatraea saccharalis & $40 \mathrm{par} / \mathrm{ha}$ \\
$\mathrm{T} 3$ & Diatraea saccharalis & $50 \mathrm{par} / \mathrm{ha}$ \\
$\mathrm{T} 4$ & Galleria mellonella & $40 \mathrm{par} / \mathrm{ha}$ \\
\hline
\end{tabular}

\section{Evaluaciones de Parasitismo}

Se realizaron evaluaciones de parasitismo antes de iniciar con las liberaciones de Billaea claripalpis a la edad del $6^{\circ}$ mes de cultivo, luego se realizaron evaluaciones de parasitismo cada 30 días (mes $7^{\circ}, 8^{\circ}$, $\left.9^{\circ}, 10^{\circ}, 11^{\circ}\right)$ y al momento de la cosecha.

Según Vinasco (1991), se tomaron una muestra de 120 tallos/ cuartel, distribuidas en zigzag, luego se procedió con las evaluaciones de parasitismo que consistieron en rajar la caña, es decir, retirando con un machete la corteza en dos franjas longitudinales a lo largo del tallo, posteriormente contar las formas biológicas de Diatraea saccharalis parasitadas por Billaea claripalpis del total de formas biológicas de Diatraea saccharalis, según la siguiente fórmula:

$$
\begin{gathered}
\text { Parasitismo }(\%)=\frac{N^{\circ} \text { formas biológicas del parasitoide }}{N^{\circ} \text { formas biológicas del parasitoide }+ \text { plaga }} \times 100 \\
\text { Infestación }(\%)=\frac{N^{\circ} \text { de cañas barreneadas }}{\text { Total de cañas }} \times 100
\end{gathered}
$$$$
\text { Intensidad Infestación }(\%)=\frac{N^{\circ} \text { de entrenudos barreneadas }}{\text { Total de entrenudos }} \times 100
$$

\section{Determinación de regresión y la correlación entre el porcentaje de intensidad de infestación y el parasitismo}

Se realizó un modelo de regresión lineal simple entre el porcentaje de intensidad de infestación y el parasitismo, para determinar el grado de asociación entre el Diatraea saccharalis y Billaea claripalpis.

\section{Análisis estadístico}

Se efectuaron cuatro repeticiones por cada tratamiento, con un diseño factorial utilizando el Softwarrd Estadístico Info Stat, versión 1.1., para realizar el Análisis de Varianza, prueba Tukey, con una probabilidad de 0.05 . 


\section{RESULTADOS Y DISCUSIÓN}

El daño que causa Diatraea saccharalis, es en su estado larval, atacan los brotes jóvenes, barrena los tallos, ocasionando pérdidas económicas a la industria azucarera. Es por eso que se debe de tener un adecuado control biológico, que debe incluir los monitoreos, liberaciones de enemigos naturales reproducidos en laboratorio y saneamiento que ayude a controlar la infestación del barrenador. (Ludeña, V. 2014). En vista de ello, de realizar un aporte en la eficacia de los parasitoides liberados en campo, la presente investigación se enfocó en determinar el efecto del parasitismo en campo de Billaea claripalpis sobre Diatraea saccharalis, criadas en su huésped natural Diatraea saccharalis y el huésped artificial Galleria mellonella, El análisis de varianza muestra diferencias significativas entre los tratamientos (tabla 3) y se muestra la agrupación del análisis de Tukey al 95\% de confianza (tabla 4), siendo el tratamiento T1, Billaea claripalpis criadas en Galleria mellonella a una dosis de 50 parejas/ ha, el que tiene el mejor promedio de parasitismo (44.37\%), seguidos de los tratamientos T4, Billaea claripalpis criadas en Galleria mellonella a una dosis de 40 parejas/ ha; tratamiento T3, Billaea claripalpis criadas en Diatraea saccharalis a una dosis de 50 parejas/ ha y el tratamiento T2, Billaea claripalpis criadas en Diatraea saccharalis a una dosis de 40 parejas/ ha con promedios de parasitismo de $41.49 \%$, $39.06 \%$ y $34.16 \%$ respectivamente.

Tabla 3. Análisis de varianza sobre el efecto del parasitismo en campo de Billaea claripalpis criadas en su huésped natural Diatraea saccharalis y el huésped artificial Galleria mellonella.

\begin{tabular}{lrrrrr}
\hline $\begin{array}{l}\text { Fuente de } \\
\text { Variación }\end{array}$ & SC & GI & CM & F & p-valor \\
\hline Modelo & 4749.63 & 9 & 527.74 & 26.84 & $<0.0001$ \\
Tratamiento & 1569.76 & 3 & 523.25 & 26.61 & $<0.0001$ \\
Día & 3179.87 & 6 & 529.98 & 26.95 & $<0.0001$ \\
Error & 2005.78 & 102 & 19.66 & & \\
Total & 6755.41 & 111 & & & \\
\hline
\end{tabular}

Tabla 4. Separación de medias por medio de Tukey al 95\% de confianza, sobre el efecto del parasitismo en campo de Billaea claripalpis criadas en su huésped natural Diatraea saccharalis y el huésped artificial Galleria mellonella.

\begin{tabular}{cccccccr}
\hline Tratamientos & Medias & n & E.E. & Grupo 1 & Grupo 2 & Grupo 3 & Grupo 4 \\
\hline T1 & 44.37 & 28 & 0.84 & A & & & \\
T4 & 41.49 & 28 & 0.84 & & B & & \\
T3 & 39.06 & 28 & 0.84 & & & C & \\
T2 & 34.16 & 28 & 0.84 & & & & D \\
\hline
\end{tabular}

14

Rev. Tayacaja 2(2): 08 - 18, 2019 ISSN:2617-9156 
Las medias en común no son significativamente diferentes $(\mathrm{P}>0.05)$

En el Figura 1, muestra las evaluaciones de parasitismo desde el $6^{\circ}$ mes hasta la cosecha del cultivo de caña de azúcar. Cabe mencionar, que Billaea claripalpis criadas en su huésped artificial Galleria mellonella, tratamientos $\mathrm{T} 1$ y T4, son las obtuvieron mejores promedios de parasitación. Esto es debido probablemente a su mayor tamaño, es mejor hospedero para este propósito, siempre y cuando se sigan algunas medidas de asepsia. (Cenicaña. 1992)

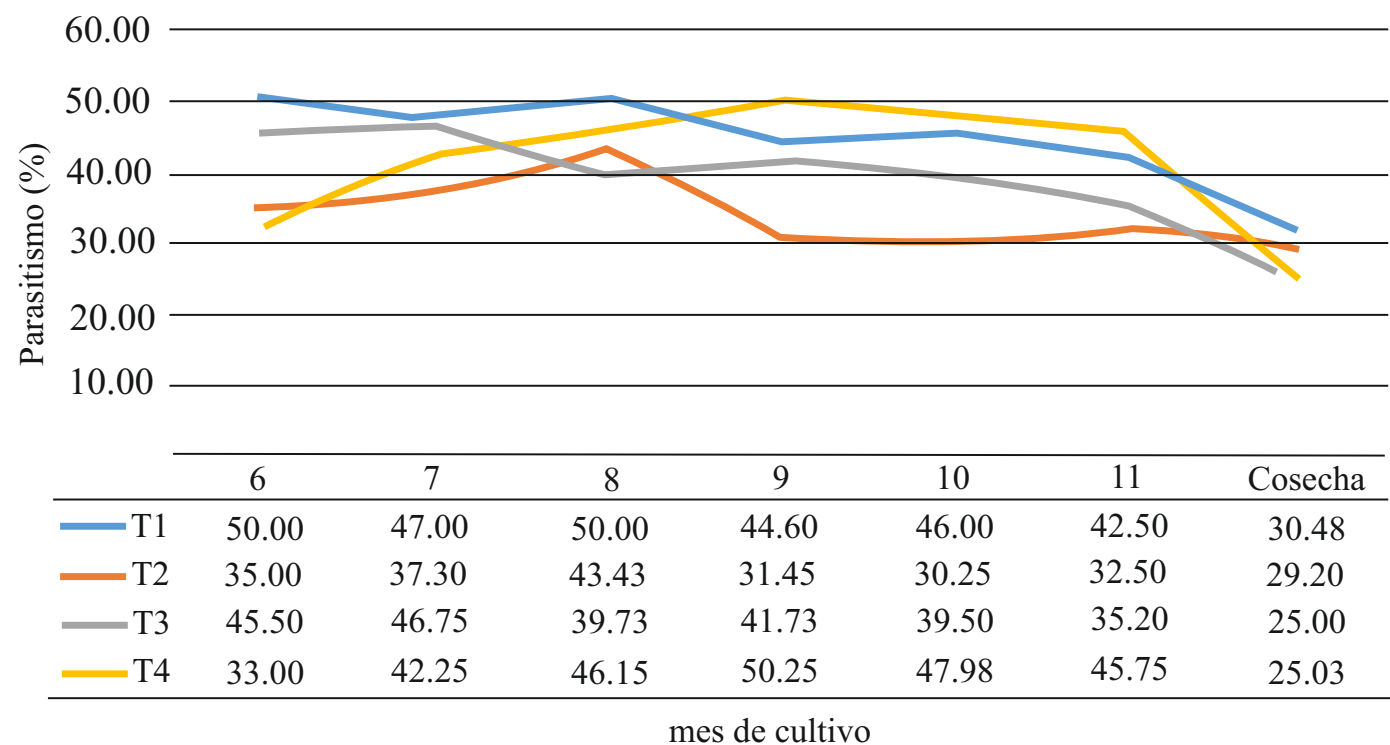

Figura1. Parasitismo de Billaea claripalpis sobre Diatraea saccharalis, según tratamientos en el cultivo de caña de azúcar

Para determinar el efecto del parasitismo de Billaea claripalpis en campo, al momento de la cosecha, se realizó evaluaciones de infestación e intensidad de infestación y parasitismo, en los diferentes tratamientos en el fundo Santa Elena I, Virú - La Libertad. 2017-2018. (tabla 5)

Tabla 5. Infestación e intensidad de infestación de Diatraea saccharalis y porcentaje de parasitismo de Billaea claripalpis al momento de la cosecha. Fundo Santa Elena I, Virú La Libertad. 2017-2018

\begin{tabular}{|c|c|c|c|}
\hline Tratamientos & $\begin{array}{c}\text { Infestación } \\
(\%)\end{array}$ & $\begin{array}{c}\text { Intensidad } \\
\text { Infestación (\%) } \\
\end{array}$ & $\begin{array}{c}\text { Parasitismo } \\
(\%)\end{array}$ \\
\hline T1 & 88.30 & 8.90 & 30.48 \\
\hline $\mathbf{T 2}$ & 85.00 & 9.30 & 29.20 \\
\hline T3 & 95.00 & 16.58 & 25.00 \\
\hline T4 & 86.67 & 14.83 & 25.03 \\
\hline
\end{tabular}


Se encontró una correlación negativa $(\mathrm{r}=$ 0.97 ) entre el parasitismo y la intensidad de infestación y $(\mathrm{r}=-0.49)$ entre el parasitismo y la infestación. El modelo que mejor nos explica los resultados de intensidad de infestación fue el cuadrático $\left(\mathrm{R}^{2}=0.9479\right)$ (figura 2). A modo que incrementa el porcentaje de control de Billaea claripalpis, decrece el porcentaje de intensidad de infestación, demostrando que este parasitoide es responsable de la disminución de los daños de Diatraea saccharalis en el fundo. Además, se determinó que la intensidad de infestación es más consistente que la infestación para correlacionar datos de la caña de azúcar y el parasitismo a nivel de campo (Badilla, et al. 1991). Esto se debe a que la intensidad de infestación, que corresponde al porcentaje de entrenudos barrenados, donde se realiza cortes longitudinales de las cañas, con lo cual los daños de los barrenadores se muestran en su totalidad, dado que a este daño se asocian con la acción de otros organismos secundarios tales como hongos y bacterias causando daños en los entrenudos siguientes. Sin embargo, en la infestación, se determina el porcentaje de cañas barrenadas, dado que no incluye el abrir las cañas.

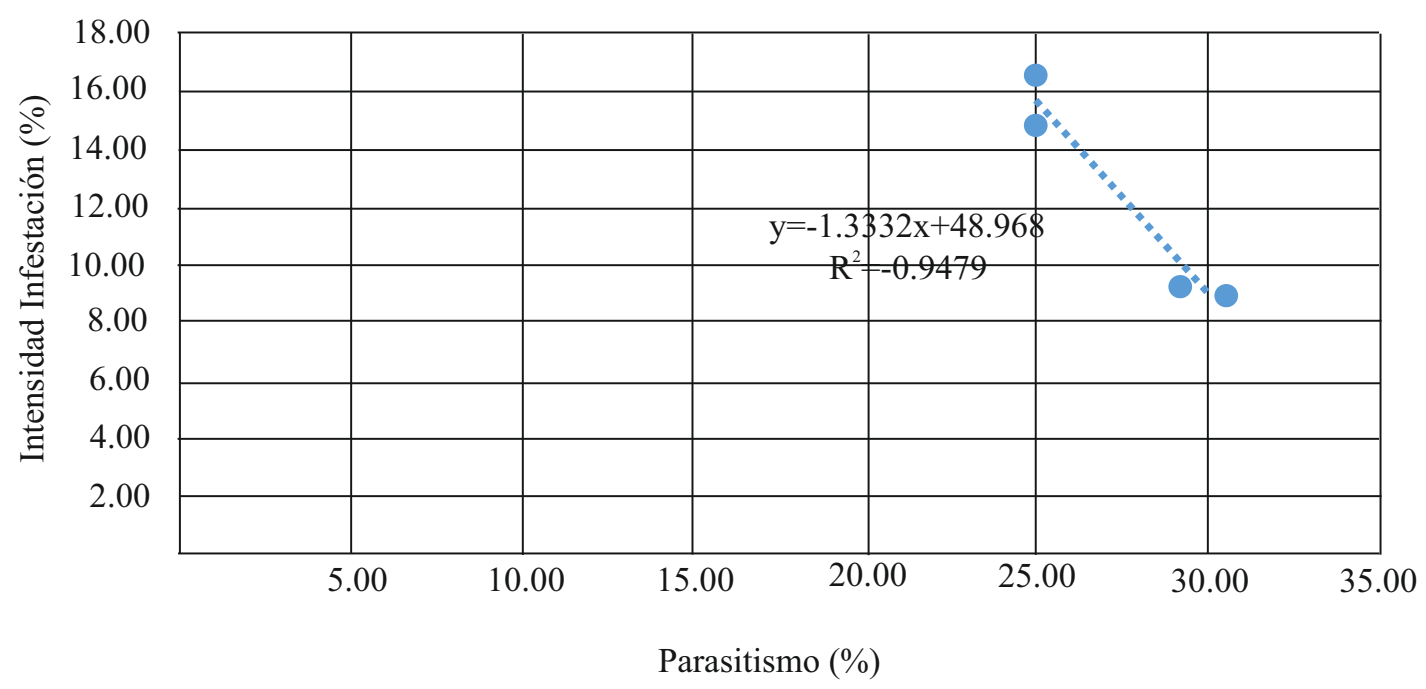

Figura 2. Correlación entre parasitismo y la intensidad infestación fundo Santa Elena I, Virú - La Libertad. 2017-2018

\section{CONCLUSIONES}

Billaea claripalpis criadas en su huésped artificial Galleria mellonella, son las que obtuvieron mejores promedios de parasitación sobre Diatraea saccharalis. La relación del porcentaje de intensidad de infestación es inversamente proporcional al porcentaje de parasitismo, con un coeficiente de correlación de $\mathrm{r}=$ 0.97 y con $\mathrm{R}^{2}=0.9479$.

Es importante evaluar a nivel de laboratorio los diferentes agentes potenciales de control biológico, antes 
antes de iniciar un programa de masivo debido a la alta especificidad en parasitoides y agentes entomopatógenos.

Diatraea saccharalis y la caña de azúcar, forman parte de un ecosistema dinámico, cambiante $\mathrm{y}$, por lo tanto, requiere de constantes investigaciones con la finalidad de reducir los niveles de daño de la plaga.

\section{REFERENCIAS BIBLIOGRÁFICAS}

Ayquipa, G. y J. Sirlopú. (1978). Crianza masiva de Diatraea saccharalis Fabr. En la dieta artificial para la propagación de su parasite Paratheresia claripalpis Wulp. Rev. Per. Ent. 21 (1): 55-56.

Badilla, F; Alfaro, D; Solis, AI; Madriz, T; Salazar, JA. (1991). Utilización de modelos de regresión para determinar el comportamiento del parasitoide Cotesia flavipes (Hymenoptera: Braconidae) en el control de Diatraea tabernella en la Hacienda Juan Viñas, Costa Rica. In Congreso de Tecnología Azucarera de Centroamérica (9, 1991, San José, Costa Rica). Resúmenes. 7p.

Bustillo, A. (2009). Acciones para reducir las poblaciones de Diatraea. Carta Trimestral, Colombia. Disponible en: https://www.cenicana.org/pdf_privado/ carta_trimestral/ct2009/ct3y4_09/ct3y4 _09_p10-15.pdf

Cadena, P.( 2008). Caracterización morfológica y molecular de especies de Diatraea spp. (Lepidoptera:
Crambidae). Convenio Colciencias Cenicaña. Disponible en: http://www.cenicana.org/pdf/no_cla sificacion/6194.pdf

Calderón, C. (1986). Cuatro dietas artificiales para la crianza masiva de Diatraea saccharalis Fabr. Rev. Per. Ent. 29:51-54

Castillo, C. (2011). Efecto comparativo de cuatro dietas artificiales en el crecimiento y desarrollo larval de Galleria mellonella L. Tesis para optar el Título Profesional de Biólogo. Fac. de Cs. Biológicas. Universidad Nacional de Trujillo. Perú.

Cenicaña, (1992). Informe Anual. Programa de variedades.

Cornejo, E. (2010). Estudio d ela biología de la Diatraea saccharalis Fabricius del cultivo de caña de azúcar, en condiciones de laboratorio en Tumbes, 2010. Tesis Agrónomo. Universidad Nacional de Tumbes. 10-32.

Goodman, R. (2003). This Agricultural Note was developed by Department of Environment and Primary Industries.

Limo, S. (2009). Incorporación de Nitrógeno al cultivo de Maíz, utilizando en la preparación de dieta artificial para larvas de Diatraea saccharalis. Tesis para optar el Título Profesional de Biólogo. Fac. de Cs. Biológicas. Universidad Nacional de 
Trujillo. Perú. 71pp.

Long, w.H.; Hensley, S.D. (1972). Insect pest of sugarcane. Ann. Rev. Entomol.(USA). (17): 149-176p.

Ludeña, V. (2014). Produccion intensiva de Diatraea saccharalis en dieta artificial, para difusion de sus parasitos Cotesia flavipes y Billaea claripalpis. Universidad de Guayaquil. Facultad de Ciencias Naturales. Escuela de Biologia. Guayaquil. Ecuador. 34p.

Mostacero, M. (2003). Eficiencia de cuatro dietas artificiales para la crianza masiva de Diatraea saccharalis Fabr. Facultad de Ciencias Biológicas. Escuela Académico Profesional de Ciencias Biológicas. Universidad Nacional de Trujillo. Trujillo. Perú

Neira, M. (2006). Sanidad apícola, principales enfermedades y enemigos de las abejas en Chile. Universidad Austral de Chile. Valdivia, Chile. 138p.

Pollack, M. y G. Ayquipa. (1992). Técnica de parasitación masiva de larvas de Diatraea saccharalis con las larvas de Paratheresia claripalpis. Rev. Per. Ent. 35: 9-10

Risco, S. (1960). La situación actual de los barrenadores de la caña de azúcar del género Diatraea y otros taladradores, en el Perú, Panamá y Ecuador. Revista Peruana de Entomología Agrícola. 3:6-10.
Risco, S., N. Morales y G. Ayquipa. (1973). Una dieta artificial para la crianza masiva de orugas del borer de la caña de azúcar Diatraea saccharalis Fabr. (Lep.: Cambidae) Saccharum ICIA 1(1): 27-42.

Rodríguez, L. (2015). Ciclo biológico de Galleria mllonella L. (Lepidoptera: Pyralidae). Universidad Nacional Agraria La Molina. 2015 . D i s p o n ible e n : http://repositorio.lamolina.edu.pe/ handle/UNALM/2107

Salvatore, A.; López, G. y E. Willink. (2009). Plagas en el cultivo de caña de azúcar, pp.143-150. Manual del Cañero. Editorial Zafra S.A. - John Deere. Tucumán, Argentina.

Vinasco A., J. (1991). Evaluación de algunos factores que inciden en la determinación del caño causado por Diatraea (Lepidoptera: Pyralidae) en caña de azúcar (Saccharum sp.). Armenia, Universidad del Quindío, Instituto de Educación a Distancia. 39 pp. (Trabajo de Grado, Tecnólogo Agropecuario)

\section{CORRESPONDENCIA}

M.Sc. Charles Frank Saldaña Chafloque charles.saldana.chafloque@gmail.com 